\title{
PREVALENCIA DE Babesia caballi, Theileria equi Y TRIPANOSOMIASIS Y ANÁLISIS DE FACTORES DE RIESGO EN EQUINOS DE ANTIOQUIA, COLOMBIA
}

\section{PREVALENCE OF Babesia caballi, Theileria equi AND TRYPANOSOMIASIS AND ANALYSIS OF RISK FACTORS IN HORSES OF ANTIOQUIA, COLOMBIA}

\author{
Alejandro Strauch ${ }^{1}$, Viviana Castillo Vanegas ${ }^{2}$, Diego Piedrahita ${ }^{3}$, Jenny Chaparro ${ }^{4}$, David Villar ${ }^{5}$, Alfredo Sánchez ${ }^{6}$, \\ Julio Tobón ${ }^{7}$, Diego Ortiz Ortega ${ }^{8}$, Nicolás Ramírez ${ }^{9}$, Martha Olivera-Ángel $^{10}$
}

${ }^{1}$ Zoot, Ms. Empresa Colombiana de Productos Veterinarios -VECOL-, Av. El Dorado No. 82-93, Bogotá-Colombia, e-mail: alejandrostrauch@hotmail.com, (DDhttps://orcid.org/0000-0001-6175-2570; ${ }^{2}$ MV, Ms. Vitalab Diagnóstico Veterinario SAS, Ciudadela Complex Lote 55 vía Llanogrande, Rionegro Antioquia, e-mail: viviana.castillo@udea.edu.co, (iDhttps://orcid. org/0000-0003-2047-7220; 3MV, Ms, Dr. Sci., Grupo de investigación CIBAV, Facultad de Ciencias Agrarias. Universidad de Antioquia, Carrera 75 No.65-87, Medellín Colombia, e-mail: diego.piedrahita@udea.edu.co, (iDhttps://orcid.org/0000-00022705-1321; 4MV, Ms, Dr. Sci.,Grupo de investigación Vericel, Escuela de Medicina Veterinaria, Facultad de Ciencias Agrarias. Universidad de Antioquia, Calle 70 No.52-21, Medellín, Colombia, e- mail: jenny.chaparro@udea.edu.co; ${ }^{5}$ MV, Ms, Ph.D, Grupo de investigación Vericel, Escuela de Medicina Veterinaria, Facultad de Ciencias Agrarias. Universidad de Antioquia, Calle 70 No. 52-21, Medellín, Colombia, e-mail: davidvillar2003@yahoo.com; ${ }^{6}$ MV, Ms, Ph.D. Empresa Colombiana de Productos Veterinarios -VECOL-, Av. El dorado No. 82 - 93, Bogotá, Colombia, e-mail: Alfredo.Sanchez@vecol.com.co; ${ }^{7}$ Admin. Agrop, Ms. Empresa Colombiana de Productos Veterinarios -VECOL-, Av. El dorado No. 82 - 93, Bogotá, Colombia, e-mail: julio. tobon@vecol.com.co, (iDhttps://orcid.org/0000-0003-2705-3770; ${ }^{8}$ MV, Ms, Ph.D. CORPOICA, Km 14 Vía Mosquera, Bogotá, Colombia, e-mail: dortiz@corpoica.org.co; ${ }^{9} \mathrm{MV}$, Ms, Dr. Sci., Grupo Centauro, Escuela de Medicina Veterinaria, Facultad de Ciencias Agrarias. Universidad de Antioquia, Calle 70 No. 52-21, Medellín, Colombia, e-mail: nicolas.ramirez@udea.edu.co; ${ }^{10}$ DMV, Dr. Sci. Agr. Grupo de investigación Biogénesis, Facultad de Ciencias Agrarias. Universidad de Antioquia, Carrera 75 No.65-87, Medellín, Colombia, e-mail: martha.olivera@udea.edu.co, iDhttps://orcid.org/0000-0001-7853-4406

Rev. U.D.C.A Act. \& Div. Cient. 21(2), 491-500, Julio-Diciembre, 2018

https://doi.org/10.31910/rudca.v21.n2.2018.976

\begin{abstract}
Artículo de acceso abierto publicado por Revista U.D.C.A Actualidad \& Divulgación Científica bajo una licencia Creative Commons CC BY-NC 4.0
\end{abstract}

\section{RESUMEN}

Esta investigación, se llevó a cabo, debido a la poca información nacional existente en hemoparásitos equinos y su objetivo fue determinar la prevalencia de babesiosis y tripanosomiasis, en el Valle de Aburrá y Rionegro, municipios de Antioquia (Colombia), así como algunos factores de riesgo asociados a la presentación de seropositividad, a estas enfermedades. En 223 predios, con una población de 1.008 equinos, se tomó muestra de sangre venosa, para realizar el diagnóstico serológico y molecular. La información epidemiológica fue obtenida, a través de la aplicación de una encuesta dirigida a las personas a cargo de los animales. Para el análisis estadístico, se aplicó Chi cuadrado de independencia y la Prueba Exacta de Fisher, cuando fue necesario. Para las asociaciones bivariadas, se calcularon estimaciones del riesgo (OR) por variable explicativa, con sus respectivos intervalos de confianza, del 95\% (I.C. 95\%). Se encontró una prevalencia del $11,9 \%$, para babesiosis y de $1,9 \%$, para tripanosomiasis; como factor de protección, se encontró el hecho de salir a una feria. Para la piroplasmosis, el lugar geográfico, el sexo, estar castrado, ser positivos a Anemia Infecciosa Equina (AIE), estrongílidos u oxiurus fueron factores de riesgo. Para tripanosomiasis, el factor de riesgo fue estar infestado con Dermacentor nitens o ser mular. Se deben reforzar métodos de vigilancia epidemiológica activa, sobre todo, en casos de movilización continua de equinos o desarrollo de eventos, que involucren presencia masiva de ejemplares.

Palabras clave: Mulas, burros, condición corporal, vigilancia activa. 


\section{ABSTRACT}

This research was carried out due to the little existing national information on equine hemoparasites; the objective was to determine babesiosis and trypanosomiasis prevalence in the Aburrá Valley and Rionegro Antioquia (Colombia) as well as some risk factors associated with the seropositivitie of these diseases. In 223 lots with a population of 1.008 equine venous blood sample was taken for serological diagnosis and molecular. Epidemiological information was obtained through the application of a survey addressed to persons in charge of the animals. For the statistical analysis, Chi square of independence or Fisher's Exact Test was applied when necessary. For the bivariate associations, risk estimates (OR) were calculated by explanatory variable, with their respective $95 \%$ confidence intervals $(95 \% \mathrm{CI})$. It was found a prevalence of $11.9 \%$ for babesiosis and $1.9 \%$ for trypanosomiasis and as protection factor found the fact of going to trade fairs. Geographic location, sex and being neutered, be positive for Equine Infectious Anemia (AIE), strongyles or oxiurus was a risk factor for piroplasmosis. For Trypanosomiasis the risk factor was to be infested with ticks Dermacentor nitens or be mule. Methods of active epidemiological surveillance should be reinforced, especially in cases of continuous mobilization of equines or development of events that involve massive presence of horses.

Keywords: mules, donkeys, body condition, active surveillance.

\section{INTRODUCCIÓN}

La piroplasmosis equina (PE) es una infección producida por uno de dos protozoos, Theileria equi o Babesia caballi, transmitidas por garrapatas o mediante jeringas contaminadas o transfusiones de sangre. La infección se ha reportado como endémica en América Central y del Sur (Guidi et al. 2015) y es de reporte sanitario obligatorio, pues afecta el movimiento transfronterizo e intrarregional de los equinos (Instituto Colombiano Agropecuario, 2015).

En Argentina, se considera endémica (Flores et al. 2013), mientras que, en Brasil, ha sido estudiada su prevalencia, especialmente, de Babesia equi (Braga et al. 2017). En Colombia, se encuentra un reporte de Calderón y colaboradores, en 2013, en Babesia sp. y, en Venezuela, se encontró, tanto B. caballi como B. equi (De Vera et al. 2006) La tripanosomiasis equina, introducida en Latinoamérica en el siglo XV, con caballos árabes por parte de los españoles (Desquesnes et al. 2013), es producida por el Trypanosoma evansi, un protozoo monomórfico extracelular de transmisión mecánica, por efecto de insectos picadores. La literatura latinoamericana reciente no reporta nuevos hallazgos; sin embargo, Desquesnes et al. 2013 presuponen que es un parásito endémico, que se propaga silenciosamente.
En Colombia, según el censo equino de 2014, existían 1.451 .085 ejemplares equinos, entre caballares, mulares y asnales, de los cuales, el 12.024 (10,61\%) de esta población, se encuentra en el departamento de Antioquia (Instituto Colombiano Agropecuario, 2014).

La cadena equina en Colombia, según el Ministerio de Agricultura (https://sioc.minagricultura.gov.co/Equino/Documentos/2014), está dividida en productores pequeños y medianos y participa con un $4,35 \%$ al PIB agropecuario y al PIB total, con el $0,1 \%$, reflejado en el consumo que el subsector hace sobre diferentes industrias, como alimentos e insumos, marroquinera, farmacéuticos, talabartería, entre otros y genera 159.752 empleos directos. El mercado nacional, se mueve entre ferias y exposiciones, subastas y ventas directas, así como sacrificio para consumo.

Debido a la importancia de la cadena equina y siendo la piroplasmosis una enfermedad que restringe la movilización nacional e internacional de los equinos y la tripanosomiasis una enfermedad zoonótica, este trabajo tuvo como objetivo determinar la prevalencia los dos agentes, así como los factores de riesgo asociados, en una población de equinos, en Antioquia, Colombia.

\section{MATERIALES Y MÉTODOS}

El estudio, se realizó en 223 predios, ubicados en los municipios que componen el área metropolitana del Valle de Aburrá (Barbosa, Girardota, Copacabana, Bello, Medellín, Envigado, Itagüí, Sabaneta, La Estrella y Caldas) y el municipio de Rionegro, localizado en el oriente antioqueño. Para el cálculo del tamaño de muestra, se tomó la población total de équidos, según el censo del ICA, previamente mencionado e información suministrada por las Secretarías de Agricultura municipales. Se usó un nivel de confianza del $95 \%$, una prevalencia esperada del $50 \%$ y un error aceptado de $2,96 \%$ ( $\leq 5 \%)$. El tamaño de la muestra fue de 1.008 animales, basado en la fracción de muestreo calculada del 8,36\%, que fue aplicada a la población de equinos de cada vereda, dentro de los municipios. Para la toma de muestra de sangre total, se tuvo acceso a 985 equinos.

Aspectos éticos: Este trabajo fue autorizado por el CEEA de la Universidad de Antioquia, acta N 100 de 26 de noviembre de 2015.

Toma de muestras y pruebas de laboratorio: Las muestras de sangre, se tomaron entre septiembre y noviembre de 2015, de la vena yugular de los animales estudiados, se procesaron en el Laboratorio de Biología Molecular de la Facultad de Ciencias Agrarias, de la Universidad de Antioquia. El aislamiento del DNA, se hizo en sangre total, en tubos 
citratados, usando el kit para sangre (QIAGEN Inc., Valencia, CA, USA) y siguiendo las instrucciones del fabricante. Cada muestra de DNA se diluyó $1: 100$ y se congeló a $-20^{\circ} \mathrm{C}$, hasta su uso. Para la detección del tripanosoma, se siguió el protocolo descrito por Masiga et al. (1992) y para Theileria equi y Babesia caballi, el de Alhassan et al. (2005), donde se usó un juego de cebadores universal, con productos de PCR de 913 bp y 867 bp, respectivamente, siendo reportado el resultado positivo como piroplasmosis, teniendo en cuenta que la diferenciación entre géneros es apenas de 46 pb y no se diferencian en el gel de agarosa usado. Cada muestra se corrió en duplicado y los oligonucleótidos usados se muestran en la tabla 1. Los productos de PCR fueron visualizados en geles de agarosa al 1,5\% y teñidos con GelRed (Biotium, USA).

Encuesta e información: Se realizaron encuestas, entrevistando a propietarios, inquilinos y administradores de los predios, incluidos en el estudio. Las entrevistas, las realizaron personas pertenecientes a instituciones gubernamentales, con experiencia en producción equina, capacitadas previamente. La encuesta aplicada está disponible a solicitud, que debe ser tramitada al autor senior del artículo.

Tabla 1. Primers de Oligonucleótidos usados en la amplificación de PCR.

\begin{tabular}{|c|c|c|c|c|}
\hline Parásito & Primer nombre & Primer secuencia & $\begin{array}{l}\text { Amplicon } \\
\text { tamaño }\end{array}$ & Referencia \\
\hline Trypanosoma & $\begin{array}{l}\text { TBR1-F } \\
\text { TBR2-R }\end{array}$ & $\begin{array}{l}\text { 5'-GAATATTAAACAATGCGCAG-3' } \\
\text { 5'-CCATTTATTAGCTTTGTTGC-3' }^{\prime} \text { '-C }\end{array}$ & $164 \mathrm{bp}$ & Masiga et al. 1992 \\
\hline Theileria equi & $\begin{array}{l}\text { BecUF1-F } \\
\text { BecUR-R }\end{array}$ & $\begin{array}{l}\text { 5'-GTTGATCCTGCCAGTAGTCA -3' } \\
5^{\prime} \text { '-CGGTATCTGATCGTCTTCGA -3' }\end{array}$ & 913 bp & Alhassan et al. 2005 \\
\hline Babesia caballi & $\begin{array}{l}\text { BecUF1-F } \\
\text { BecUR-R }\end{array}$ & $\begin{array}{l}\text { 5'-GTTGATCCTGCCAGTAGTCA -3' } \\
5^{\prime} \text {-CGGTATCTGATCGTCTTCGA -3' }\end{array}$ & 867 bp & Alhassan et al. 2005 \\
\hline
\end{tabular}

Medición de la condición corporal: Se realizó acorde con De Vera et al. (2006), de 1 a 5, donde 1 es muy flaco, 2 flaco, 3 ideal, 4 carnoso, 5 gordo.

Tipo de estudio: Se efectuó un estudio observacional analítico de corte transversal.

Análisis de los datos: En el análisis descriptivo, se utilizaron distribuciones absolutas y relativas. Se aplicó la prueba Chi cuadrado de independencia o la Prueba Exacta de Fisher, cuando fue necesario. Para estudiar asociaciones bivariadas, se calcularon estimaciones del riesgo (OR), por variable explicativa, con sus respectivos intervalos de confianza del $95 \%$ (I.C. 95\%). Para determinar los factores de riesgo asociados a la infección, se ajustó un modelo multinivel de respuesta binaria; la variable respuesta fue la presencia o no del parasito ( $\mathrm{Si}, \mathrm{No})$. Los dos modelos ajustados (piroplasmosis, tripanosomiasis) tienen dos niveles: el primero es relativo al individuo y el segundo son los municipios de estancia de los equinos. El procesamiento y análisis de los datos, se realizó mediante el programa estadístico R versión 3.3.1.

Análisis multivariado: La selección de las variables incluidas en los modelos explicativos, se hizo mediante el criterio de Hosmer-Lemeshow (valores $\mathrm{p}<0.25$ ), así: Piroplasmosis: sexo, grupo etario, condición corporal, salida a ferias, AIE, estrongílidos, Strongylus vulgaris, oxiuriasis equina. Tripanosomiasis: Especie, garrapatas.
En el análisis multinivel, se excluyó el municipio de Itagüí, ya que en este municipio solo se registró un individuo. Después de excluir registros con datos faltantes, en total, se consideraron 618 equinos, en el modelo de piroplasmosis y 984, en el de tripanosomiasis. Antes de ajustar los modelos multinivel, se constató la significancia estadística del parámetro de varianza, estimado en el modelo multinivel nulo. Se probó la hipótesis nula , mediante el estadístico de razón de verosimilitud. En los dos modelos ajustados (piroplasmosis, tripanosomiasis), se observaron valores $p$ menores a 0,05 , lo que sugiere que el modelo propuesto es adecuado.

\section{RESULTADOS Y DISCUSIÓN}

En la tabla 2, se muestran los animales positivos a piroplasmosis, en el que se evidencia una población positiva del 12,33\% ( $\mathrm{n}=114)$, de los cuales, 110 animales pertenecen a la especie caballar, lo que confirma lo encontrado por Calderón et al., quienes, en 2013, encontraron una prevalencia de $18,25 \%$, en equinos del departamento de Córdoba. Para el caso de Trypanosomiasis, se encontró una incidencia del $1,96 \%(n=19)$, donde 15 animales son equinos y 4 mulares, contrastando con lo reportado por Salim et al. (2013), quienes reportaron en Sudán, una prevalencia de animales positivos del $12 \%$, en caballares y $3,4 \%$, en asnales, no muestrearon mulares. 
Tabla 2. Animales positivos a Piroplasmosis y Tripanosomiasis, según especie.

\begin{tabular}{|l|l|c|c|c|}
\hline & Especie & Negativo (\%) & Positivo (\%) & Total \\
\hline & Caballar & $814(88,1)$ & $110(11,9)$ & 924 \\
\hline Piroplasmosis & Mular & $45(93,8)$ & $3(6,2)$ & 48 \\
\hline & Asnal & $12(92,3)$ & $1(7,7)$ & 13 \\
\hline & Total & 871 & 64 & 985 \\
\hline & Caballar & $909(98,4)$ & $15(1,6)$ & 924 \\
\hline Trypanosomiasis & Mular & $44(91,7)$ & $4(8,3)$ & 48 \\
\hline & Asnal & 13 & & 13 \\
\hline & Total & 966 & $19(1,96 \%)$ & 985 \\
\hline
\end{tabular}
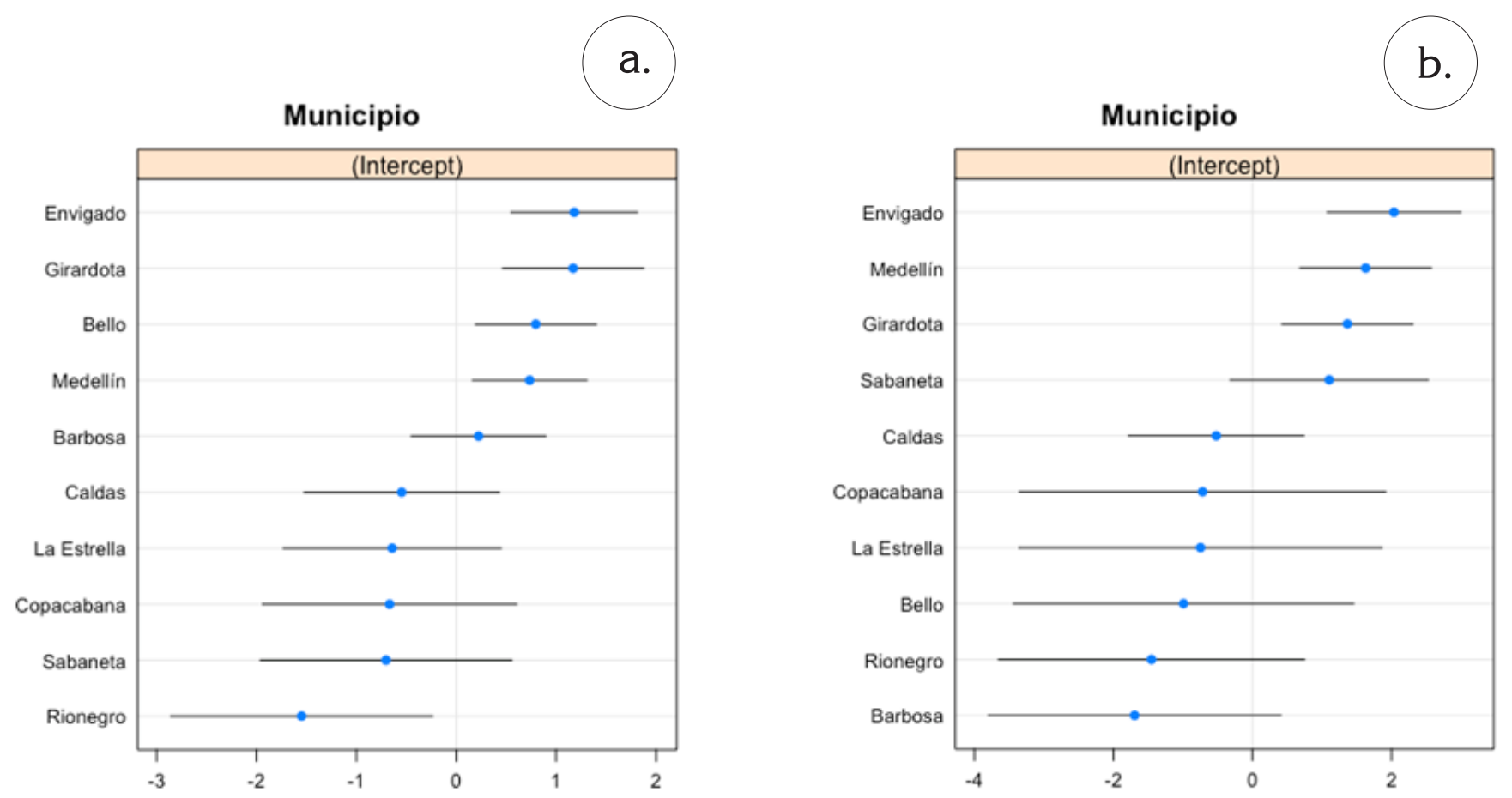

Figura 1. Análisis de OR en diferentes municipios para la infección en equinos de: a. Piroplasmosis; b. Tripanosomiasis. Valores de $\mathrm{OR} \geq 1$ es indicativo de ser un factor de riesgo, por su parte un $\mathrm{OR} \leq 1$ indica un factor protector. por su parte un $\mathrm{OR} \leq 1$ indica un factor protector.

En la figura 1a., se observa que, en los municipios Envigado, Girardota, Bello y Medellín, se encontró un riesgo significativamente mayor de aparición de piroplasmosis, mientras que en el municipio de Rionegro, un riesgo significativamente menor. El municipio de Rionegro, se encuentra a $2.080 \mathrm{~m}$ s.n.m., mientras que los municipios anteriormente mencionados, la altura oscila entre 1.300 y $2.800 \mathrm{~m}$ s.n.m. y temperaturas que oscilan entre 13 y $24^{\circ} \mathrm{C}$, factores que favorecen la presencia de garrapatas, artrópodos vectores de los hemoparásitos.

Para tripanosomiasis, se observó que los municipios de Envigado, Medellín y Girardota tienen un riesgo mayor de infección (Figura 1b); esto se puede deber a la cercanía de estos municipios y al continúo transporte de todo tipo de semovientes, dentro del valle del Aburrá. 
En este estudio, se pudo demostrar que existen animales infectados por hemoparásitos, aunque en bajas proporciones (Tabla 2), esto significa que existe riesgo de infección para otros animales. Se sugiere seguir lo que ordena la autoridad sanitaria colombiana ICA y el código sanitario para animales terrestres (capítulo 4.16) (OIE, 2016), con el fin de prevenir su diseminación, entre animales que se muevan de una re- gión a otra o que participan en eventos ecuestres y generan riesgo epidemiológico.

Entre los équidos fueron los caballares los que presentaron más animales positivos (Tabla 2), siendo el lugar de estancia del animal lo que influye en su positividad (Tabla 3), con menor riesgo en los machos que las hembras $(\mathrm{OR}<1)$ (Tabla 4); es de anotar que los machos capones son los que presentan menor riesgo de infección a piroplasmosis (Tabla 4 y 5).

Tabla 3. Distribución absoluta y porcentual de las características demográficas, la condición corporal, salidas del animal a ferias y resultados de pruebas de laboratorio incluidas en el estudio, en 11 municipios de Antioquia, Colombia, 2017.

\begin{tabular}{|c|c|c|c|c|c|c|c|c|c|c|c|}
\hline Variable & Categoría & $\begin{array}{c}\text { Barbosa } \\
\text { n (\%) }\end{array}$ & $\begin{array}{l}\text { Bello } \\
\text { n (\%) }\end{array}$ & $\begin{array}{c}\text { Caldas } \\
\text { n (\%) }\end{array}$ & $\begin{array}{c}\text { Copaca- } \\
\text { bana } \\
\text { n (\%) }\end{array}$ & $\begin{array}{c}\text { Envigado } \\
\text { n (\%) }\end{array}$ & $\begin{array}{c}\text { Girardota } \\
\text { n (\%) }\end{array}$ & $\begin{array}{c}\text { La } \\
\text { Estrella } \\
\text { n (\%) }\end{array}$ & $\begin{array}{l}\text { Medellín } \\
\text { n (\%) }\end{array}$ & $\begin{array}{c}\text { Sabaneta } \\
\text { n (\%) }\end{array}$ & $\begin{array}{c}\text { Rionegro } \\
\text { n (\%) }\end{array}$ \\
\hline \multirow{3}{*}{ Sexo } & Hembra & $114(69,9)$ & $42(58,3)$ & $116(65,9)$ & $32(72,7)$ & $32(56,1)$ & $66(77,6)$ & $31(52,5)$ & $42(55,3)$ & $29(50,9)$ & $117(53,7)$ \\
\hline & $\begin{array}{l}\text { Macho } \\
\text { entero }\end{array}$ & $16(9,8)$ & $25(34,7)$ & $27(15,3)$ & $3(6,8)$ & $13(22,8)$ & $14(16,5)$ & $17(28,8)$ & $13(17,1)$ & $17(29,8)$ & $67(30,7)$ \\
\hline & $\begin{array}{l}\text { Macho } \\
\text { capón }\end{array}$ & $33(20,2)$ & $5(6,9)$ & $33(18,8)$ & $9(20,5)$ & $12(21,1)$ & $5(5,9)$ & $11(18,6)$ & $21(27,6)$ & $11(19,3)$ & $34(15,6)$ \\
\hline \multirow{3}{*}{ Especie } & Caballar & $139(85,3)$ & $66(91,7)$ & 169 (96) & $39(88,6)$ & $55(96,5)$ & $80(94,1)$ & $51(86.4)$ & $71(93.4)$ & $54(94,7)$ & $215(98,6)$ \\
\hline & Mular & $22(13,5)$ & $4(5,6)$ & $6(3,4)$ & $5(11,4)$ & $2(3,5)$ & $5(5,9)$ & $1(1,7)$ & $5(6,6)$ & $1(1,8)$ & $1(0,5)$ \\
\hline & Asnal & $2(1,2)$ & $2(2,8)$ & $1(0,6)$ & - & - & - & $7(11,9)$ & - & $2(3,5)$ & $2(0,9)$ \\
\hline \multirow{5}{*}{$\begin{array}{l}\text { Grupo } \\
\text { etario } \\
\text { (meses) }\end{array}$} & $\begin{array}{l}\text { Menos } \\
\text { de } 6\end{array}$ & $5(3,1)$ & $1(1,4)$ & $5(2,8)$ & $1(2,3)$ & - & $4(4,7)$ & - & - & - & $6(2,8)$ \\
\hline & 6 a 12 & $4(2,5)$ & $5(6,9)$ & $8(4,5)$ & $1(2,3)$ & $1(1,8)$ & - & - & $1(1,3)$ & $1(1,8)$ & $3(1,4)$ \\
\hline & 12 a 48 & $49(30,1)$ & $32(44,4)$ & $44(25)$ & $10(22,7)$ & $13(22,8)$ & $21(24,7)$ & $21(35,6)$ & $16(21,1)$ & $15(26,3)$ & $102(46,8)$ \\
\hline & 48 a 96 & $58(35,6)$ & $21(29.2)$ & $53(30,1)$ & 20 (45.5) & $20(35.1)$ & $43(50,6)$ & $20(33,9)$ & $36(47.4)$ & 25 (43.9) & $39(17,9)$ \\
\hline & Más de 96 & $47(28,8)$ & $13(18,1)$ & $66(37,5)$ & $12(27,3)$ & $23(40,4)$ & $17(20)$ & 18 (30.5) & $23(30,3)$ & $16(28,1)$ & $68(31,2)$ \\
\hline \multirow{5}{*}{$\begin{array}{l}\text { Condición } \\
\text { corporal }\end{array}$} & 1 & $1(0,6)$ & - & - & $1(2.3)$ & - & - & $1(1,7)$ & - & - & - \\
\hline & 2 & $26(16,1)$ & - & $9(5,1)$ & $1(2,3)$ & - & - & - & $1(1,3)$ & $3(5,3)$ & $10(4,6)$ \\
\hline & 3 & $122(75,8)$ & $32(44,4)$ & $48(27,3)$ & $14(32,6)$ & $8(14)$ & $65(81,3)$ & $13(22)$ & $10(13,3)$ & $19(33,3)$ & $122(56,5)$ \\
\hline & 4 & $12(7,5)$ & $40(55.6)$ & $77(43,8)$ & $27(62.8)$ & $38(66.7)$ & $15(18,8)$ & $44(74,6)$ & $34(45.3)$ & 30 (52.6) & $77(35,6)$ \\
\hline & 5 & - & - & $42(23,9)$ & - & $11(19.3)$ & - & $1(1,7)$ & $30(40)$ & $5(8,8)$ & $7(3,2)$ \\
\hline \multirow{2}{*}{$\begin{array}{l}\text { Sale a } \\
\text { feria }\end{array}$} & No & $160(98,8)$ & $72(100)$ & $165(95,4)$ & 43 (100) & $57(100)$ & 85 (100) & 59 (100) & $75(98,7)$ & 57 (100) & $214(99,5)$ \\
\hline & Sí & $2(1,2)$ & - & $8(4,6)$ & - & - & - & - & $1(1,3)$ & - & $1(0,5)$ \\
\hline
\end{tabular}


Continuación Tabla 3.

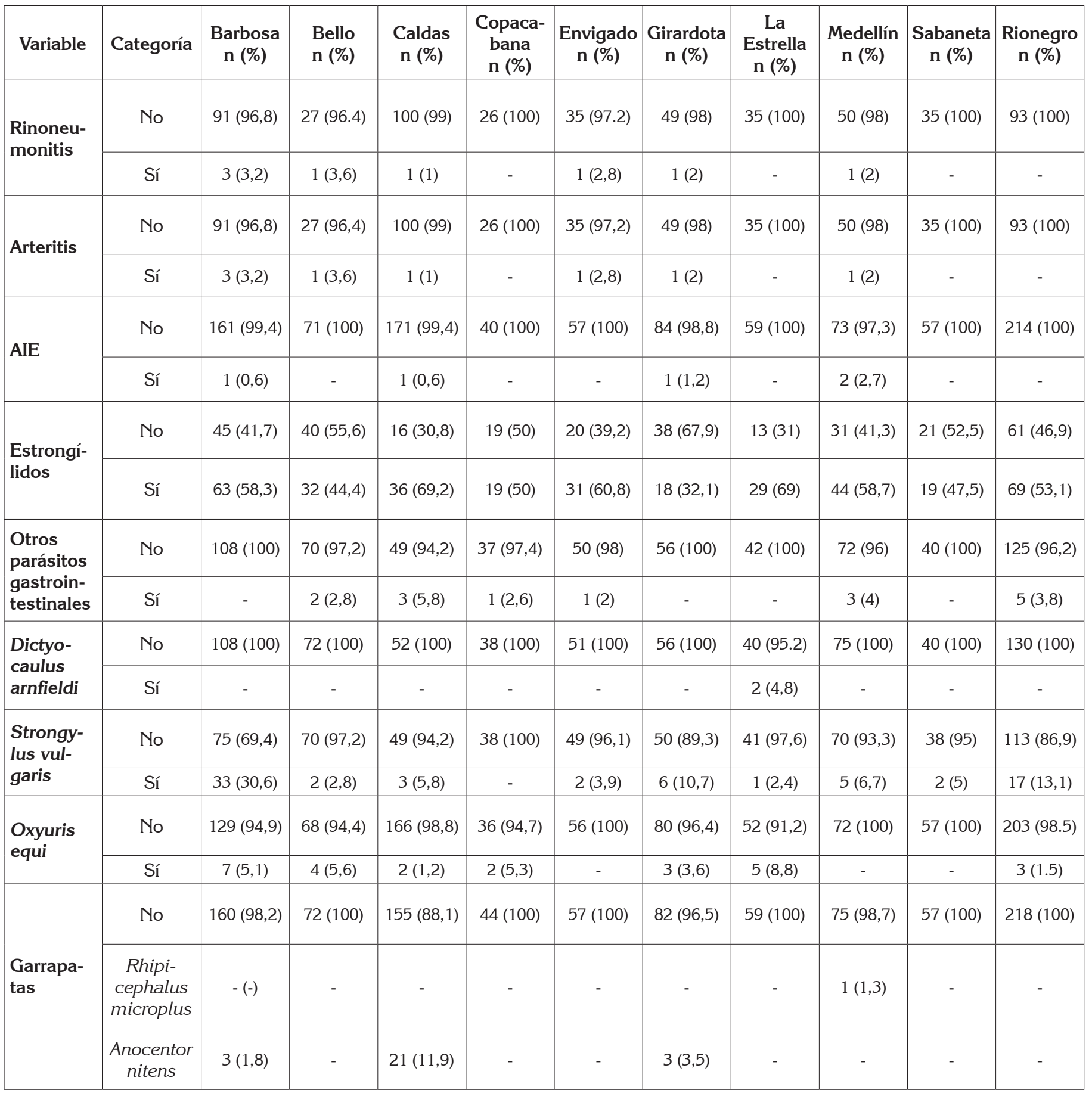


Tabla 4. Asociación de piroplasmosis, tripanosomiasis, con variables demográficas y pruebas de laboratorio, así como con el lugar de estancia, en once municipios de Antioquia, Colombia, 2017.

\begin{tabular}{|c|c|c|c|}
\hline \multirow{2}{*}{ Variable } & \multirow{2}{*}{ Categoría } & Piroplasmosis & Tripanosomiasis \\
\hline & & OR (IC 95\%) & OR (IC 95\%) \\
\hline \multirow{3}{*}{ Sexo } & Hembra & 1 & 1 \\
\hline & Macho entero & $0,93(0,57 ; 1,51)$ & $0,39(0,09 ; 1,74)$ \\
\hline & Macho capón & $0,57(0,31 ; 1,05)$ & $0,47(0,11 ; 2,06)$ \\
\hline \multirow{3}{*}{ Especie } & Caballar & 1 & 1 \\
\hline & Mular & $0,49(0,15 ; 1,61)$ & $5,51(1.76,17.29)$ \\
\hline & Asnal & $0,62(0,08 ; 4,79)$ & - \\
\hline \multirow{5}{*}{ Grupo etario (meses) } & Menos de 6 & 1 & - \\
\hline & 6 a 12 & - & - \\
\hline & 12 a 48 & $0,48(0,13 ; 1,74)$ & 1 \\
\hline & 48 a 96 & $1,08(0,31 ; 3,81)$ & $2,92(0.78,10.87)$ \\
\hline & Más de 96 & $0,87(0,24 ; 3,08)$ & $2,45(0.63,9.57)$ \\
\hline \multirow{5}{*}{ Condición corporal } & 1 & - & - \\
\hline & 2 & 1 & - \\
\hline & 3 & $1,82(0,55 ; 6,1)$ & 1 \\
\hline & 4 & $2,02(0,6 ; 6,77)$ & $1,59(0,63 ; 4)$ \\
\hline & 5 & $3,41(0,95 ; 12,28)$ & - \\
\hline \multirow{2}{*}{ Sale a feria } & No & 1 & 1 \\
\hline & Sí & $0,16(0,07 ; 0,37)$ & $0,61(0,18 ; 2,13)$ \\
\hline \multirow{2}{*}{ Rinoneumonitis } & No & 1 & 1 \\
\hline & Sí & $0,69(0,09 ; 5.4)$ & - \\
\hline \multirow{2}{*}{ Arteritis } & No & 1 & 1 \\
\hline & Sí & $0,91(0,11 ; 7,49)$ & - \\
\hline \multirow[b]{2}{*}{ AIE } & No & 1 & 1 \\
\hline & Sí & $11,69(1,93 ; 70,72)$ & - \\
\hline \multirow{2}{*}{ Estrongílidos } & No & 1 & 1 \\
\hline & Sí & $1,55(0,93 ; 2,61)$ & $1,13(0,39 ; 3,29)$ \\
\hline \multirow{2}{*}{ Otros parásitos gastrointestinales } & No & 1 & 1 \\
\hline & Sí & $0,59(0,08 ; 4,59)$ & - \\
\hline \multirow{2}{*}{ Dictyocaulus arnfieldi } & No & 1 & 1 \\
\hline & Sí & - & - \\
\hline \multirow{2}{*}{ Strongylus vulgaris } & No & 1 & 1 \\
\hline & Sí & $0,47(0,17 ; 1,33)$ & $1,37(0,3 ; 6,24)$ \\
\hline \multirow{2}{*}{ Oxyuris equi } & No & 1 & 1 \\
\hline & Sí & $2,52(0,98 ; 6,48)$ & $2,14(0.27,16.76)$ \\
\hline \multirow{3}{*}{ Garrapatas } & No & 1 & 1 \\
\hline & Rhipicephalus microplus & - & - \\
\hline & Anocentor nitens & $1,34(0,45 ; 3,94)$ & $4,42(0,97 ; 20,19)$ \\
\hline
\end{tabular}


Tabla 5. Factores de riesgo asociados a la piroplasmosis, tripanosomiasis en equinos, con lugar de estancia, en diez municipios de Antioquia, Colombia.

\begin{tabular}{|c|c|c|c|c|}
\hline \multirow{2}{*}{ Variables } & \multicolumn{2}{|c|}{ Piroplasmosis } & \multicolumn{2}{|c|}{ Tripanosomiasis } \\
\hline & OR & IC $95 \%$ & OR & IC $95 \%$ \\
\hline Intercepto & 0,01 & $0 ; 0,08$ & 001 & $0 ; 0,04$ \\
\hline \multicolumn{5}{|l|}{ Variables individuales } \\
\hline Sexo (Macho entero/Hembra ${ }^{a}$ ) & 1,26 & 0,$63 ; 2,52$ & - & - \\
\hline Sexo (Macho capón/Hembra a) & 0,43 & 0,$18 ; 1,03$ & - & - \\
\hline Especie( Mular/Caballar ${ }^{\mathrm{a}}$ ) & - & - & 7,6 & 2,$14 ; 26,92$ \\
\hline Especie (Asnal/Caballar ${ }^{\mathrm{a}}$ ) & - & - & - & - \\
\hline Grupo etario & 1,36 & 0,$96 ; 1,92$ & - & - \\
\hline Condición corporal & 1,39 & 0,$88 ; 2,19$ & - & - \\
\hline Sale a feria $\left(\mathrm{S} i / \mathrm{No}^{\mathrm{a}}\right)$ & 0,3 & 0,$11 ; 0,84$ & - & - \\
\hline Arteritis (Sí/No $\left.{ }^{a}\right)$ & & & - & - \\
\hline $\operatorname{AIE}\left(\mathrm{Si} / \mathrm{No}^{\mathrm{a}}\right)$ & 2,15 & 0,$17 ; 27,42$ & - & - \\
\hline Estrongílidos (Sí/ $\left./ \mathrm{No}^{\mathrm{a}}\right)$ & 1,82 & 1,$03 ; 3.23$ & - & - \\
\hline Strongylus vulgaris (Sí/No $\left.{ }^{a}\right)$ & 0,61 & 0,$2 ; 1,87$ & - & - \\
\hline Oxyuris equi (Sí/No $\left.{ }^{a}\right)$ & 3,32 & 0,$92 ; 12,02$ & - & - \\
\hline Garrapatas (Rhipicephalus microplus/No ${ }^{\mathrm{a}}$ ) & - & - & - & - \\
\hline $\begin{array}{l}\text { Garrapatas } \\
\text { (Anocentor nitens/No }{ }^{\mathrm{a}} \text { ) }\end{array}$ & - & - & 10,34 & 1,$61 ; 66,52$ \\
\hline \multicolumn{5}{|l|}{ Variable nivel 2} \\
\hline- & - & - & - & - \\
\hline Varianza nivel 2 (Donde se encuentra) & \multicolumn{2}{|r|}{1,12} & \multicolumn{2}{|r|}{3,08} \\
\hline CPV & \multicolumn{2}{|r|}{$25,5 \%$} & \multicolumn{2}{|r|}{$48,4 \%$} \\
\hline
\end{tabular}

${ }^{\text {a }}$. Nivel de referencia.

* En cada caso se ajustó un Modelo multinivel de respuesta binaria, intercepto aleatorios y efectos fijos.

Como se deduce de la tabla 5, los animales que salen a feria tienen menos riesgos de infección (93 a 63\% menos riesgo), ya que con ellos se tienen mayores cuidados, se desparasitan, se alimentan y se acicalan, previo a la presentación en feria. Las variables como positividad a AIE, estróngilidos o a oxiuros (Tabla 5), son un factor de riesgo en la presentación de piroplasmosis, lo que concuerda con que animales que no se desparasitan de rutina o presentan una enfermedad consuntiva, como la Anemia Infecciosa, son más susceptibles de presentación de enfermedad.

Es importante mencionar que, aunque las garrapatas son los agentes transmisores de piroplasmosis y pueden transmitir la tripanosomiasis de los équidos, solo se encontraron 28/64 caballos con garrapatas (no se muestran los datos) y de estos solamente 4 fueron positivos para piroplasmosis y dos, para tripanosomiasis, por lo que se infiere que existen otras formas de transmisión. Es interesante anotar el hecho de que los animales que salen a ferias están protegidos contra todos los hemoparásitos (Tabla 4), aparentemente por el cuidado permanente y las exigencias de las ferias para el ingreso de los animales.

En cuanto a la tripanosomiasis son las mulas las que presentan mayor riesgo de infección $(\mathrm{OR}=5,51)$, entre los équidos (Tabla 4), así como los equinos que tienen Dermacentor nitens, $(\mathrm{OR}=4,42)$ (Tabla 4).

Los resultados contrastan con la literatura nacional, en donde reportan, en el departamento de Córdoba, mayores nive- 
les de Babesia spp. (18,25\%) (Calderón et al. 2013). En Brasil, se encontró $50,4 \%$ de positividad a $T$. equi (Ferreira et al. 2016)2016; en la India (Sumbria et al. 2016)2016 reportaron 14,14 y $0,0 \%$, respectivamente, a $T$. equi y a $B$. caballi); en Italia, 70,3\% (185/263) positivos, a T. equi y 10,3\% (27/263), a B. caballi (Del Pino et al. 2016)2016 y en Francia, T. equi y B. caballi, que mostraron seroprevalencias del $58 \%$ y $12,9 \%$, respectivamente (Guidi et al. 2015). Es difícil comparar los resultados con esta literatura, ya que, aunque este trabajo hizo el estudio molecular en $T$. equi y $B$. caballi, no se realizaron reportes individuales de cada uno de los agentes, sino la positividad a piroplasmosis (ver metodología); sin embargo, las prevalencias de uno u otro parásito están por encima de lo reportado en este trabajo.

Con respecto a la tripanosomiasis, se puede decir que la prevalencia es muy baja, ya que apenas un 1,9\% de animales fueron positivos, con mayor presencia en caballares que en mulares, lo mismo encontrado por Salim et al., en el 2013. Es importante resaltar que ningún asnal fue positivo, resultados similares a lo encontrado en India, por Sumbria et al. (2014), pero mucho más baja a la registrada en Sudán, que fue el 12,7\% (Salim et al. 2013). La recomendación en ambos casos es que se debe alertar a los servicios veterinarios y que el país realice estudios epidemiológicos de la enfermedad y adopte estrategias de control.

Los hemoparásitos son una amenaza silenciosa, puesto que los animales infectados no presentan signos sino cuando la enfermedad se encuentra en un estado avanzado, es por esto, que se destaca la necesidad de continuar con la vigilancia activa de piroplasmosis y tripanosomiasis, para reportarlas al ICA y tomar las medidas pertinentes, acorde con la OIE.

Agradecimientos: A la Universidad de Antioquia, a la Unidad de Diagnóstico de la Facultad de Ciencias Agrarias. Conflicto de intereses: El manuscrito fue preparado y revisado con la participación de todos los autores, quienes declaramos que no existe conflicto de intereses que ponga en riesgo la validez de los resultados presentados.

\section{REFERENCIAS}

1. ALHASSAN, A.; PUMIDONMING, W.; OKAMURA, M.; HIRATA, H.; BATTSETSEG, B.; FUJISAKI, K.; YOKOYAMA, N.; IGARASHI, I. 2005. Development of a single-round and multiplex PCR method for the simultaneous detection of Babesia caballi and Babesia equi in horse blood. Vet. Parasitol. (Netherlands). 129(1-2):43-49.

2. BRAGA, M.D.S.C.O.; COSTA, F.N.; GOMES, D.R.M.; XAVIER, D.R.; ANDRÉ, M.R.; GONÇALVES, L.R.; FRESCHI, C.R.; MACHADO, R.Z. 2017. Genetic diversity of piroplasmids species in equids from island of São Luís, northeastern Brazil. Rev. Bras. Parasitol Vet. 26(3):331-339.

3. CALDERÓN, A.; CARDONA, J.; VERGARA, Ó. 2013. Frequency of Babesia spp. in horses of Monteria, Cordoba (Colombia). Rev. U.D.C.A Act. \& Div. Cient. 16(2):451-458.

4. DE VERA, M.; GUILLÉN, A.T.; GARCÍA, F.; CONTRERAS, R.; SIERRALTA, Á.; LEÓN, E. 2006. Seroprevalencia de la babesiosis equina en caballos purasangre de carrera alojados en los hipódromos La Rinconada y Nacional de Valencia, Venezuela. Veterinaria Trop. 31(1):2.

5. DEL PINO, L.E.B.; ROBERTO, N.; VINCENZO, V.; FRANCESCA, I.; ANTONELLA, C.; LUCA, A.G.; FRANCESCO, B; TERESA, S.M. 2016. Babesia caballi and Theileria equi infections in horses in Central-Southern Italy: Sero-molecular survey and associated risk factors. Ticks Tick Borne Dis. (Netherlands). 7(3):462-469.

6. DESQUESNES, M.; HOLZMULLER, P.; LAI, D.-H.; DARGANTES, A.; LUN, Z.-R.; JITTAPLAPONG, S. 2013. Trypanosoma evansi and surra: a review and perspectives on origin, history, distribution, taxonomy, morphology, hosts, and pathogenic effects. BioMed Res. Int. Disponible desde Internet en: https://www. hindawi.com/journals/bmri/2013/194176/ ～(con acceso 15/12/2017).

7. FERREIRA, E.P.; VIDOTTO, O.; ALMEIDA, J.C.; RIBEIRO, L.P.; BORGES, M.V.; PEQUENO, W.H.; STIPP, D.T.; DE OLIVEIRA, C.J.; BIONDO, A.W.; VIEIRA, T.S. 2016. Serological and molecular detection of Theileria equi in sport horses of northeastern Brazil. Comp Immunol Microbiol Infect Dis. (England). 47:72-76.

8. FLORES, D.A.; MINICHIELLO, Y.; ARAUJO, F.R.; SHKAP, V.; BENIITEZ, D.; ECHAIDE, I.; ROLLS, P.; MOSQUEDA, J.; PACHECO, G.M.; PETTERSON, M.; FLORIN-CHRISTENSEN, M.; SCHNITTGER, L. 2013. Evidence for extensive genetic diversity and substructuring of the Babesia bovis metapopulation. Transbound Emerg Dis. 60 Suppl 2:131-6.

9. GUIDI, E.; PRADIER, S.; LEBERT, I.; LEBLOND, A. 2015. Piroplasmosis in an endemic area: analysis of the risk factors and their implications in the control of Theileriosis and Babesiosis in horses. Parasitol Res. (Germany). 114(1):71-83. 
10. INSTITUTO COLOMBIANO AGROPECUARIO, ICA. 2014. Censo Equinos 2013. Disponible desde Internet en: https:/www.ica.gov.co/getdoc/bfb8ddc3-70364b85-aa79-6fa98b8614eb/Censo-Equinos-2008. aspx (con acceso 10/12/2017).

11. INSTITUTO COLOMBIANO AGROPECUARIO, ICA. 2015. Resolución 003714 de 2015. Establecer la declaración obligatoria ante el ICA, de las enfermedades, infecciones e infestaciones. Artículo 4. Bogotá, Colombia.

12. MASIGA, D.K.; SMYTH, A.J.; HAYES, P.; BROMIDGE, T.J.; GIBSON, W.C. 1992. Sensitive detection of trypanosomes in tsetse flies by DNA amplification. Int. J. Parasitol. (Australia). 22(7):909-918.

13. OIE. O.M. D. L.S.A. 2016. Código sanitario para animales terrestres. Disponible desde Internet en: http://www. oie.int/es/normas-internacionales/codigo-terrestre/ acceso-en-linea/?htmfile $=$ chapitre_high_level.htm (con acceso 14/12/2017).
14. SALIM, B.; BAKHEIT, M.A.; SUGIMOTO, C. 2013. Molecular detection of equine trypanosomes in the Sudan. Vet Parasitol. (Netherlands). 200(3):246-250.

15. SUMBRIA, D.; SINGLA, L.; SHARMA, A.; MOUDGIL, A.D.; BAL, M. 2014. Equine trypanosomosis in central and western Punjab: Prevalence, haematobiochemical response and associated risk factors. Acta Trop. (Netherlands). 138:44-50.

16. SUMBRIA, D.; SINGLA, L.D.; SHARMA, A. 2016. Theileria equi and Babesia caballi infection of equids in Punjab, India: a serological and molecular survey. Trop. Anim. Health. Prod. (Netherlands). 48(1):45-52.

Recibido: Enero 18 de 2018

Aceptado: Septiembre 5 de 2018

Cómo citar:

Strauch, A.; Castillo Vanegas, V.; Piedrahita, D.; Chaparro, J.; Villar, D.; Sánchez, A.; Tobón, J.; Ortiz Ortega, D.; Ramírez, N.; Olivera-Ángel, M. 2018. Prevalencia de Babesia caballi, Theileria equi y tripanosomiasis y análisis de factores de riesgo en equinos de Antioquia, Colombia. Rev. U.D.C.A Act. \& Div. Cient. 21(2):491-500. https://doi.org/10.31910/rudca.v21. n2.2018.976 